\title{
Applying XCS Model to Spread Trading of Taiwan Stock Index Futures
}

\author{
Jung-Bin Li, Shih-Chuan Fu, and An-Pin Chen ${ }^{1}$ \\ ${ }^{1}$ Institute of Information Management, National Chiao Tung University
}

\begin{abstract}
This study attempts to find the possibility of making relatively higher profit with lower risk when trading futures commodities. The system applies XCS classifiers to explore the rules of spread trading of these commodities. Our simulation holds a trading strategy that in every transaction, the proposed model buys and sells the same lots of goods of Taiwan index futures. All trades are settled by the end of each trading day.

The outcome of this study shows that all the proposed three trading strategies that utilize XCS outperform spread trading decisions made by traditional buy low sell high strategy during the testing period. Regarding to the issue of profitability, intraday trading by XCS also has better performance than the control group. Hence this proposed shows its value in assisting investors to have extra reward without bearing higher risks.
\end{abstract}

Keywords: spread trading, eXtended Classifier System, intraday trading

\section{Introduction}

The original purposes of serving futures products are price discovery and risk transfer. However, futures are commonly applied in operations of hedging and arbitrage. The product price is decided by the majority of investors in practice. If the market is affected by speculative investors for abnormal issues, irrational responses will be revealed from the market. Later investors adopt conservative policy to balance the potential risk, and such behavior will lead the market price back to a reasonable level. If there is sufficient difference between spot and futures prices, speculators would make arbitrage trading to earn profits. Eventually the market price goes back to a place that the majority of investors regard arbitrage as not profitable. Namely the basis between spot and futures prices is returning to a fair level.

The proposed model applies XCS classifiers to explore the rules of market behavior for spread trading with the futures commodities in Taiwan.

\section{Literature Review}

\subsection{Basis-related studies}

Studies have shown the profitability of trades based on the basis. Merrick (1987) found a significant one-way relationship between volume of spot trade and opportunities for speculation in NYSE, which explains that when there are opportunities for speculation, investors will make transactions for expected profits. In this case, the trade volume will be expanded. Marilyn and Robert (1998) consider trade volume not only reflects the supply-demand equilibrium but also an indicator of market liquidity, especially the futures. Bessembinder and Seguim (1993) suggest a substantial correlation between volume and price volatility.

Wang and Yau (2000) reveal negative correlation of volume and basis, and positive correlation of volume and open interest. It implies that the increase of trade volume helps reflect the relation between price of futures commodity and that of physical, and effectively diminish their basis and raise the market efficiency.

\section{2 eXtended Classifier System}

$\mathrm{XCS}$ is an adaptive rule-based system, which adopts the methodology of dynamic learning. Wilson (1995) proposed XCS to build a learning mechanism with payoff to increase accuracy and improve the conventional way of learning. XCS reacts to the dynamic exterior information, and it can also help resolve the most opportune solution for multi-variable problems. 


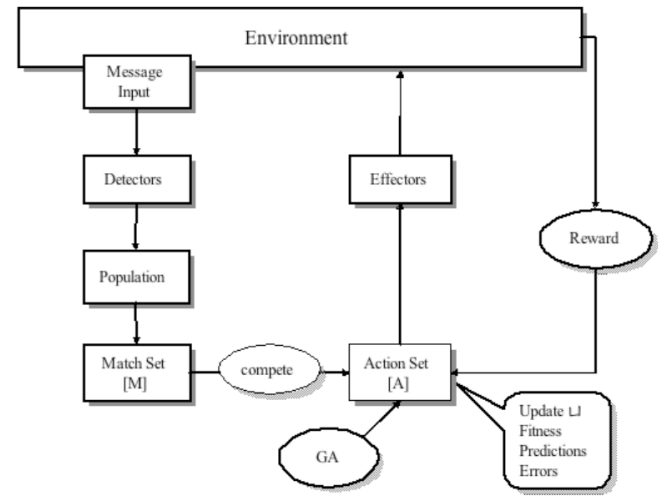

Fig.1 XCS architecture

The architecture of XCS is illustrated in Fig.1. The XCS operation model is described as follows:

[1] Receive market information and encode it as Message.

[2] If there is no such Message in Rule Base, then Detector generates a new Message and add it into Rule Base.

[3] Message is compared with the Rule Base. If there is a match, a MatchSet[M] is collected.

[4] Test the strongest ActionSet[A] in market according to the fitness and prediction values, and collect the rewards.

[5] If a match cannot be found after a number of messages are compared, genetic evolution or mutation is adopted to deal with the changes in stock market.

[6] Feed the reward back to all rules in ActionSet[A].

[7] Repeat steps [1] to [6].

\subsection{Financial applications of XCS}

Beltrametti, Fiorentini, Marengo, and Tamborini (1997) apply Classifier System to the interaction of USD-DM and USD-JPY in foreign exchange market. This study shows the response of Classifier System towards dynamic changes of the exterior environment. Rules are induced from various system environments for concluding the most appropriate solution.

Liao and Chen's study (2001) applies LCS and technical analysis indices to build a dynamic trading strategy learning model and shows a superior percentage of accuracy than random walk, and also higher accumulated profitability than buy-and-hold strategy. Similar conclusion is induced by Schulendburg and Ross (2002) in their empirical study on the variance of IBM stock price. The
LCS-based trade decisions have better profitability than random walk and buy-and-hold strategies.

\section{Model architecture}

This study trains the proposed model with three futures products: Taiwan Stock Index Futures (FITX), Taiwan Financial Index Futures (FITF), and Taiwan Electronics Index Futures (FITE). Hereinafter, they are also expressed as TX, TF, and TE for short. We attempt to find the best trading combination via this empirical study, and confirm the model's good profitability in basis transactions.

\subsection{Data of research}

The source of this empirical study is the per-minute transaction data from Taiwan Stock Exchange and Taiwan Futures Exchange. Sampling period is from May 2005 to Oct. 2005. We collect original data of transaction time, price, and volume, and convert it into transaction records on a per-minute base.

\subsection{Model design}

\section{Basis rate difference}

In this study, we collect data and calculate the difference of basis rates of two different commodities as model input. Eq. (1) is then taken to standardize the basis.

Standardized basis $(\mathrm{BR})=(\mathrm{P}($ Index $)-\mathrm{P}($ Futures $)) /$

$\mathrm{P}($ Index $)$

\section{Weight of trade volume}

Regarding to Bessembinder and Seguim's study of the substantial relationship about volume and price volatility, and Wiley and Daigler's similar conclusion, our model adopts index trade volume and its relative weight as model input.

The target commodities of this study are FITX, FITE, and FITF. As shown in Eq. (2), the commodity with greater weight is taken as denominator.

Specific weight $=$ Total trade volume $($ Index2) $/$ Total trade volume(Index 1)

\section{Credit assignment}

This model adopts hierarchical reward paradigm suggested in Wilson's works. Reward is the profit gained via spreading trades. This model trades out a target commodity once the possible profit is larger 
than the take-profit setting, and vice versa. Since the proposed model has a prerequisite of intra-day trading, the profit/loss computation for spreading transaction without reaching stop-loss or take-profit points in a day is done by counting the price of the final spot index transaction on that day.

\subsection{Evaluation scheme}

\section{Accuracy}

The output actions are analyzed, and this study focuses on the actions which predict profitable spreading transactions. Successful prediction in this model is defined as the result of predicting winning actually gains profit which is equal to or greater than the preset take-profit point.

After an accuracy evaluation of the three spreading trade combinations, the best strategy combination is offered by this model as a reference of investors' decisions.

The major concern to set stop-loss and take-profit points is the transaction cost. In the proposed model, stop-loss point is set to make the possible loss no more than the amount of trading cost. In this study, the cost of every transaction is assumed as NTD2200. Therefore, the maximum loss is also 2200, while the maximum profit is set 4400 to expand the revenue.

\section{Profitability}

The futures traders in Taiwan have to deposit margins, and it is an assumption of this study that all trades of the proposed model have sufficient margins to make all trades proceed. The profit of spreading transactions is calculated as Eq. (3):

Profit $=($ Contract value of selling - Contract value of buying) - (Contract value of selling - Contract value of buying) * Tax rate - Service charge * 2 .

\section{Performance evaluation}

In this study, the model is compared with a random trade strategy. This random trade operates on the fundamental "buy low sell high" principle to make transactions at random. It is more practical to "random walk" strategy which is taken by many studies on the analytical basis. The proposed model and the control group are analyzed in terms of accuracy and profitability.

\subsection{Accuracy analysis}

The successful prediction in this study is to evaluate the predicted result in testing period. We compare the three pairs of empirical strategies with each other, and with the control group as well. The testing model is analyzed by the experience value of XCS. To apply the rules induced in training session, model performance is analyzed by differentiating experience values and by picking rules with their prediction values greater than 90 .

Table 1 Rates of successful prediction of futures spread trading

\begin{tabular}{ccccccc}
\hline \hline \multirow{2}{*}{ Yr/Mth } & \multicolumn{2}{c}{ TXTE } & \multicolumn{2}{c}{ TXTF } & \multicolumn{2}{c}{ TETF } \\
\cline { 2 - 7 } & XCS & Rdm & XCS & Rdm & XCS & Rdm \\
\hline $2005 / 8$ & $92 \%$ & $34 \%$ & $81.6 \%$ & $33.3 \%$ & $93.9 \%$ & $48.3 \%$ \\
\hline $2005 / 9$ & $60.7 \%$ & $8.8 \%$ & $77.9 \%$ & $22.1 \%$ & $73.2 \%$ & $29.5 \%$ \\
\hline $2005 / 10$ & $89 \%$ & $26.5 \%$ & $76.7 \%$ & $30.1 \%$ & $82.4 \%$ & $27.5 \%$ \\
\hline \hline
\end{tabular}

Fig. 2 illustrates the model performance in August 2005. The outcome reveals remarkable superior performance of our XCS learning model than the buy low sell high control group, and similar outcomes are found in the other remaining testing months. In our empirical analysis, the best trading strategies of August, September, and October are spread trades of TETF, TXTF, and TXTE, respectively. Table 1 is the brief summary.

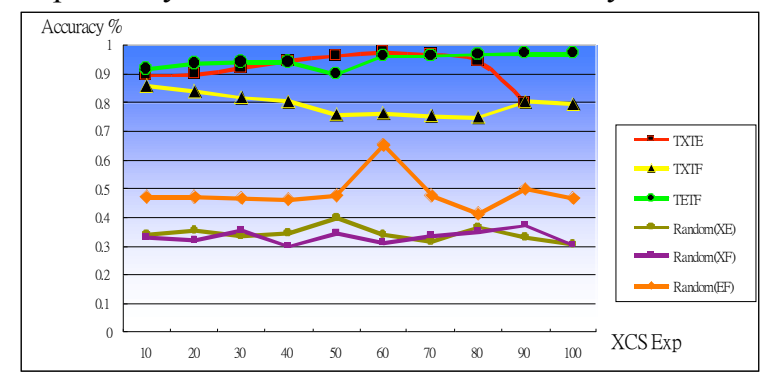

Fig 2 Rate of Successful prediction of futures spread trading in August, 2005

\subsection{Profitability analysis}

The evaluation done in this section is based on the net profit, which deducts transaction costs from the gross profit. As shown in Fig. 3, the testing model has better performance than the random trade model, and similar outcomes are found in the other remaining testing months. We analyze model performance in terms of average profit per transaction and total profit. The superior trade combination in the testing months August, September, and October are TETF, TXTE, and TXTE respectively. This outcome shows that there is no single trading strategy which always has the best performance, and our proposed model dynamically react to the environmental fluctuation. Table 2 is the brief summary. 
Table 2 Comparison of average profit of futures spreading (in thousands)

\begin{tabular}{ccccccc}
\hline \multirow{2}{*}{ Yr/Mth } & \multicolumn{2}{c}{ TXTE } & \multicolumn{2}{c}{ TXTF } & \multicolumn{2}{c}{ TETF } \\
\cline { 2 - 7 } & XCS & Rdm & XCS & Rdm & XCS & Rdm \\
\hline $2005 / 8$ & 2.03 & -1.14 & 1.74 & -1.55 & 2.46 & -0.82 \\
\hline $2005 / 9$ & 0.77 & -1.97 & 1.32 & -1.71 & 1.14 & -1.64 \\
\hline $2005 / 10$ & 2.23 & -1.66 & 1.63 & -1.95 & 1.31 & -2.51 \\
\hline \hline
\end{tabular}

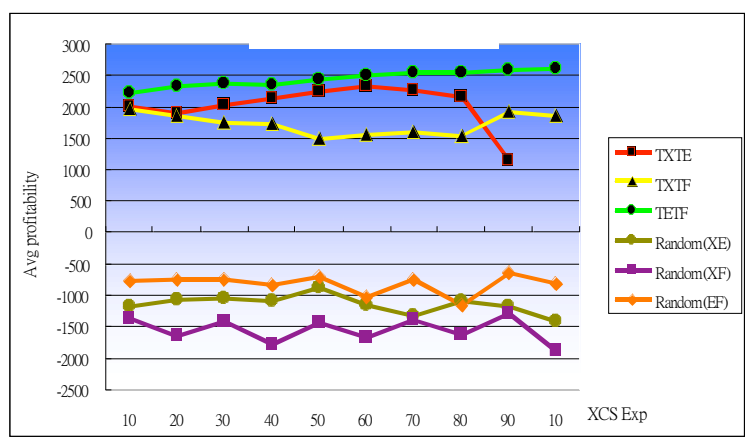

Fig 3 Futures spreading average profit of August 2005

\subsection{Summary}

In terms of accuracy, the overall average accuracy of our XCS model is $80 \%$, which is superior to that of random trade control group $(30 \%)$. The trading strategy with best accuracy is $82 \%$ by the spread trading composition of FITX and FITE. The performance of our model is also superior to the random trade model with respect to profitability. All three compositions of the proposed have profits, while the control group causes loss. Hence this proposed model shows the opportunity for making profits from intra-day spread trading. With the capability of dynamic learning of XCS, our model has remarkable performance in dealing with environmental fluctuation.

\section{Conclusion}

Regarding to the prediction accuracy and profitability, this model remarkably outperforms the random trade model, which is based on the buy low sell high basic principle. The investment strategies of the best performance in each month of the testing session are different; in other words, XCS with the capability of dynamic learning helps find a most appropriate solution for investors. Furthermore, the average profit and total profit of each month are also better. In the three-month testing session from August to October 2005, spread trading compositions with best performance are TX-TE, TX-TF, and TX-TE, respectively. In terms of total profit, the TE-TF spread trading has the best performance. The performances of the remaining strategies, in a descending order, are TX-TF, TX-TE, and lastly random trade. The outcome of this model explains that the less the relation between two objects for spread trading, the greater profit the proposed model makes, and vice versa.

The spread trading prediction model proposed is shown a useful tool to assist investment decisions. The XCS' capability of dynamic learning has been identified in our empirical study.

\section{References}

[1] Beltrametti, L., Fiorentini, R., Marengo, L., and Tamborini, R., "A Lrarning-to-Forcast Eqneriment on the Foreign Exchange Market with a Classifier System", Journal of Economic Dynamics and Control, 21, pp.1543-1575, 1997

[2] Bessembinder, H., and Seguim, P. J., "Pricing Volatility, Trading Volume, and Market Depth: Evidence from Futures Markets", Journal of Futures and Quantitative Analysis, 28, pp.21-39, 1993.

[3] Liao, P.Y., Chen, J.S., "Dynamic Trading Strategy Learning Model Using Learning Classifier Systems", Proceedings of the 2001 Congress on Evolutionary Computation, Volume 2, pages 783-789, 2001.

[4] Marilyn, K. W. and Robert, T. D., "Volume Relationships Among Types of Traders in the Financial Futures Markets," The Journal of Futures Markets, 18, pp.91-113, 1998.

[5] Merrick, J., "Volume Determination in Stock and Stock Index Futures Markets: An Analysis of Arbitrage and Volatility Effects," Journal of Futures Markets, 7, pp.483-496, 1987.

[6] Schulendburg, S. and Ross, P., "Eqnlorations in LCS Models of Stock Trading," The Fourth International Workshop on Advances in Learning Classifier Systems 2001, pp.151-180, Springer-Verlag, Berlin, 2002.

[7] Wang G. and Yau, J., “ Trading Volume, Bid-Ask Spread, and Price Volatility in Futures Markets", The Journal of Futures Markets, 20, 10, pp.943-970, 2000.

[8] Wilson, S. W., "Classifier Fitness Based on Accuracy", Evolutionary Computation, 3, 2, 1995. 